\title{
Application of 3D Printing Technology for Designing Light-weight Unmanned Aerial Vehicle Wing Structures
}

\author{
Seung Ki Moon', Yu En Tan', Jihong Hwang', and Yong-Jin Yoon ${ }^{1, \#}$ \\ 1 School of Mechanical and Aerospace Engineering, NTU Additive Manufacturing Centre, Nanyang Technological University, 50 Nanyang Avenue, Singapore 639798 \\ 2 Department of Mechanical System Design Eng., Seoul Nat'I Univ. of Sci. \& Tech., 233 Gongreung-ro, Nowon-gu, Seoul, Korea, 139-743 \\ \# Corresponding Author / E-mail: yongjiny@ntu.edu.sg, TEL: +65-6790-5033, FAX: +65-6792-4062
}

KEYWORDS: 3D Printing, Additive manufacturing, Deployable wing design, Light-weight structures, Unmanned aerial vehicles

\begin{abstract}
Unmanned Aerial Vehicles (UAVs) have been developed to perform various military and civilian applications, such as reconnaissance, attack missions, surveillance of pipelines, and interplanetary exploration. The present research is motivated by the need to develop a fast adaptable UAV design technologies for agile, fuel efficient, and flexible structures that are capable of adapting and operating in any environments. The objective of this research is to develop adaptive design technologies by investigating current design methods and knowledge of deployable technologies in the area of engineering design and manufacturing. More specifically, this research seeks to identify one truss lattice with the optimal elastic performance for deployable UAV wing design according to the Hashin \& Shtrikman theoretical bounds. We propose three lattice designs - 3D Kagome structure, 3D pyramidal structure and the hexagonal diamond structure. The proposed lattice structure designs are fabricated using an Objet $3503 \mathrm{D}$ printer while the material chosen is a polypropylene-like photopolymer called Objet DurusWhite RGD430. Based on compression testing, the proposed inflatable wing design will combine the advantages of compliant mechanisms and deployable structures to maximize flexibilities of movement in UAV design and development.
\end{abstract}

Manuscript received: March 21, 2014 / Revised: April 22, 2014 / Accepted: April 28, 2014 (Invited Paper)

\section{NOMENCLATURE}

$\begin{array}{ll}\sigma_{B} & \text { Flexural modulus } \\ \sigma_{T} & \text { Tensile strength } \\ \sigma_{Y} & \text { Yeild strength } \\ \sigma_{U} & \text { Flexural strength } \\ E & \text { Modulus of elasticity } \\ H & \text { Height } \\ \Delta & \text { Change }\end{array}$

\section{Introduction}

Unmanned Aerial Vehicles (UAVs) have been developed to perform various military and civilian applications, such as reconnaissance, attack missions, surveillance of pipelines, and interplanetary exploration. Recently, small and deployable UAVs have gained attention due to a wide range of potential applications with sophisticated operations and increased flexibilities for smaller transportation enclosures and storage. The idea of incorporating inflatable structures into flight has existed for a long time, but it was only in the last few decades that inflatable wing technology was properly developed. One of the earliest successful demonstrations was the Goodyear inflatoplane developed during the 1950s. During this period, Goodyear Aerospace designed and manufactured numerous aircraft prototypes equipped with inflatable components. One of their final designs was the GA-468 Inflatoplane. ${ }^{1}$ The inflatoplane was developed as a military rescue plane that can be dropped behind enemy lines near downed pilots to help them escape. More recently, NASA's Dryden research center developed the I2000 micro UAV that was designed with wings made of inflatable tubes surrounded with crushable foam. During its test flight, the UAV was launched from a larger UAV "mother ship" at an altitude of around 300 meters and its inflatable wings deployed from a compacted state in about one-third of a second. The wings were developed by Vertigo, Inc. for the US Navy as a gun-launched observation vehicle. Compared with the inflatoplane, the I2000 is much smaller and has a wingspan of $1.63 \mathrm{~m}$ and a chord length of $0.18 \mathrm{~m}^{2}$ By changing the wings of a UAV from a rigid 
design to an inflatable one, improved portability through reduced volume/weight ratio is one possible advantage that can be obtained. A second possible advantage of using an inflatable wing is that the geometry of the wing can be easily changed from, for example, the standard wing spans to a high-aspect ratio designs to suit mission requirements.

High-strength light-weight materials have relatively high stiffness and yield strength that are achievable at low density. They play a significant role in achieving fuel efficiency goals for the aerospace and automotive industries among others. ${ }^{3,4}$ Honeycombs, foams, and truss lattice structures are examples of the high-strength light-weight materials. The three structures have been extensively developed and optimized to provide unique performance benefits in various applications. ${ }^{5-7}$

Based on the results from literatures, it can be seen that $3 \mathrm{D}$ truss lattices are superior to both metal foams and honeycombs in terms of elastic modulus. Moreover, in contrast to closed cell metal foams and honeycombs which are open only from one direction, truss lattices has the added advantage of an open structure for multi-functional applications such as heat transfer roles. The objective of this research is to develop adaptive design technologies by investigating current design methods and knowledge of deployable technologies in the area of engineering design and manufacturing. More specifically, we investigate the apparent strengths of truss lattices to identify the truss lattice with the optimal elastic performance. We compare the compressive strength of three lattice structures that have high values in elastic performance for deployable UAV wing design. We propose three lattice designs, such as 3D Kagome structure, 3D pyramidal structure and the hexagonal diamond structure, for compression testing. The proposed lattice structure designs are fabricated using an Objet 350 3D printer while the material chosen is a polypropylene-like photopolymer called Objet DurusWhite RGD430. Based on the compression testing, the proposed inflatable wing design will combine the advantages of compliant mechanisms and deployable structures to maximize flexibilities of movement in UAV development.

The remainder of this paper is organized as follows. Section 2 reviews related literature and background in lattice structures. Section 3 describes the proposed method for fabricating the proposed three structures. Section 4 gives experiments for a compression test. Results, discussion, and applications are described in Section 5. Closing remarks and future work are presented in Section 6.

\section{Literature Reviews and Background}

\subsection{Additive Manufacturing}

Additive manufacturing (AM) is an umbrella term referring to a group of technologies used for building physical models, prototypes, patterns, tooling components, and final production parts from computer data, three dimensional scanning systems, or video games. ${ }^{8} \mathrm{AM}$ forms objects layer by layer through the joining of liquid, powder, or sheet materials as opposed to traditional manufacturing techniques like machining which are subtractive in nature. AM is used to produce parts that are difficult or impossible to fabricate using other techniques. ${ }^{9}$

Some of these technologies include stereolithography, selective laser melting (SLM), three dimensional printing (3DP), and fused deposition modeling (FDM). Each technology has its own strengths and weaknesses. The number of AM technologies is consistently growing as awareness, acceptance and application of these technologies by designers, engineers and other professionals increase.

\subsection{Structures for High-strength Light-weight Materials}

A honeycomb consists of an array of hollow cells separated by thin vertical walls. The cells are normally columnar and hexagonal in shape although rectangular and triangular shapes are also possible. A common application of honeycomb is in sandwich composite panels for use in aircraft structures. ${ }^{5}$

Metal foams are sponge-like materials that are manufactured by injecting inert gas into molten metal. The resulting structure is a solid filled with voids that have varying sizes and shape. If individual voids are fully encapsulated by the solid, the structure formed is a closed cell metal foam. Similarly, if the voids overlap and form an interconnected network, then the structure formed is an open cell metal foam. ${ }^{6}$

Lattice structures consist of repeating units of identical skeleton structures of geometric three dimensional shapes such as a polyhedron arranged in a regular pattern. New manufacturing processes have enabled lattice structures to be more easily fabricated than before. The methods devised permit entire lattice structures of unit cells ranging from millimeters to centimeters to be produced. ${ }^{7}$ The mechanical properties of honeycombs, metal foams and truss lattices have been studied extensively and numerous publications on this subject can be found. From literatures, the elastic moduli of honeycombs, metal foams, and truss lattices are compared against the Hashin-Shtrikman (HS) bounds. In others, honeycombs are compared with foams and truss lattices are compared with honeycombs etc. The HS upper bound represents the maximum values for the effective elastic moduli of isotropic two-phase composites for a given phase volume fraction. ${ }^{10}$ In 3D applications, rank-6 laminates are known to attain the HS bounds on the bulk and shear moduli. ${ }^{11}$ Rank laminates are obtained by a sequential process where at each stage the previous laminate is laminated again with a single lamina in a new direction. However, a rank laminate is a multi-length-scale structure and therefore not manufacturable. Thus, there is a need to find a single-length-scale substitute in honeycombs, foams or truss lattices with the optimal microstructure.

Among relevant research, one study indicated that closed cell foams can be superior to honeycombs with respect to shear strength and shear modulus. Also, closed cell foams provide compressive strengths which are isotropic and yet can be comparable to the compressive strengths of honeycombs in the thickness direction. ${ }^{12}$ However, the study does not indicate whether closed cell foams attain the HS upper bounds. The bulk and shear moduli of an octet-truss lattice material having singlelength-scale microstructure were evaluated against the HS upper bound and found to be about half the attainable values. ${ }^{13}$ The study also compared the stiffness and strength of the octet-truss lattice to those of metal foams and found that the lattice is 3 to 10 times stiffer.

\subsection{Description of 3D Lattice Structures}

3D Kagome lattice structure - 2D Kagome structure originated as a traditional bamboo basket weave pattern and was identified by 
topology optimization as an optimal structure based on its elastic modulus for a range of fraction volumes. ${ }^{13}$ The $3 \mathrm{D}$ variant was proposed by a recent research investigating if the superior properties observed in the $2 \mathrm{D}$ variant are carried over. ${ }^{14} 3 \mathrm{D}$ Kagome core panels were found to be superior to both tetrahedral and pyramidal truss core panels. Both analytical and empirical studies attributed the greater load carrying capacity and lower softening rate beyond the peak load to the 3D variant's diminished sensitivity to plastic buckling. ${ }^{15,16}$ In this experiment, the 3D Kagome structure is tested as a truss core panel sandwiched between two solid face sheets as shown in Fig. 1. This is identical to the configuration used in earlier experiments. ${ }^{16}$ The $3 \mathrm{D}$ Kagome structure is formed by having pairs of tetrahedrons vertically inverted and rotationally offset from each other by $60^{\circ}$.

Hexagonal diamond structure - The hexagonal diamond is a hexagonal modification of the cubic diamond structure. It has been found embedded in meteorites ${ }^{17}$ and synthesized in the laboratory using high temperatures and pressures. ${ }^{18}$ This structure may also be present in carbon films grown using chemical vapor deposition that is found to contain a high density of (111) microtwins and stacking faults.

The local atomic arrangements of the hexagonal and cubic diamond are similar. Both have covalent tetrahedral bonds and contain sixmembered rings of bonds. The main difference is the alignment of their (111) atomic layers (each pair labeled as A, B or C). In the cubic
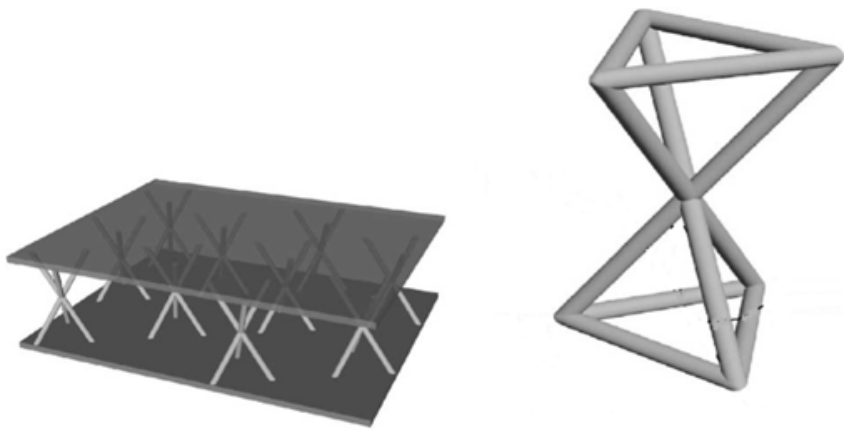

Fig. $13 \mathrm{D}$ Kagome truss core panel and unit cell of 3D Kagome structure $^{16}$

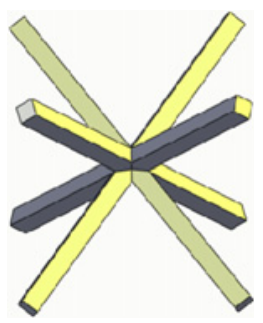

Fig. 2 Hexagonal diamond structure

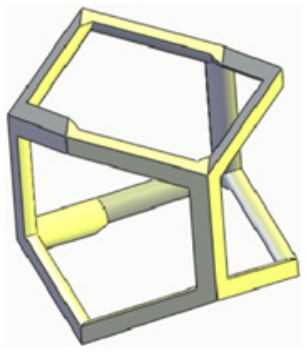

Fig. 3 Cross pyramidal structure diamond, each successive layer is displaced sideways from the previous with the fourth layer returning to the same position as the first leading to a stacking sequence $\mathrm{ABCABC} \ldots$. The stacking sequence of hexagonal diamond, in contrast, is of the type $\mathrm{ABAB}$...

More importantly, research has shown that the hexagonal diamond is around $58 \%$ harder than cubic diamond and is able to resist up to 152 GPA of indentation pressure. ${ }^{19}$ Given the unique properties of hexagonal diamond, we are interested in evaluating the performance of this structure as a macro-sized lattice structure. The unit cell structure of the hexagonal diamond is shown in Fig. 2.

Cross pyramidal structure - A pyramidal lattice structure consists of four inclined trusses meeting at a sheet node. The advantage of this design is that the incline of the trusses can be altered to support larger amount of compressive stress or shear stress depending on the requirements of the structure.

In order to obtain a design that can be more easily converted into a multi-layered structure, we modified the original design and derived an alternate structure formed by having two pyramidal structures placed against each other. The alternate design is shown in Fig. 3.

\section{3D Printing Method}

Numerous options exist for the mass production of lattice structures but they become limited for the production of one-offs or small quantities. The problem becomes even more acute for complex designs with undercuts and overhanging features since very few conventional manufacturing techniques can produce them. For testing purposes, multiple variants may be required for different geometric parameters of the design e.g. truss diameter, size of unit cell, density of structure which mean more tooling are needed.

AM offers a less expensive and more flexible design and manufacturing option as the layer-by-layer approach allows arbitrary shapes, including lattice structures, to be easily produced directly from computer-aided design (CAD) files. ${ }^{20}$ Also, the per-unit cost of an AM part is the same regardless whether the machine makes one part or hundreds of parts.

The method chosen is commercialized by Objet Geometries Ltd. It combines ink-jet technologies with the use of photopolymers as raw materials to produce 3D parts. The system chosen was the Objet 350 $3 \mathrm{D}$ printer while the photopolymer selected is a polypropylene-like material called Objet DurusWhite RGD430. Its properties are listed in Table 1.

According to at least one U.S. patent document assigned to the company, the printer works by depositing liquid photopolymer in the shape of $3 \mathrm{D}$ objects and then exposing the liquid to ultra-violet radiation to harden it. ${ }^{21-23}$

The CAD model of the various structures is prepared according to

Table 1 Properties of objet duruswhite

\begin{tabular}{cccc}
\hline & Symbol & Units & Metric \\
\hline Tensile strength & $\sigma_{T}$ & $\mathrm{MPa}$ & $20-30$ \\
\hline Modulus of elasticity & $E$ & $\mathrm{MPa}$ & $1000-1200$ \\
\hline Flexural strength & $\sigma_{U}$ & $\mathrm{MPa}$ & $30-40$ \\
\hline Flexural modulus & $\sigma_{B}$ & $\mathrm{MPa}$ & $1200-1600$ \\
\hline
\end{tabular}


standard guidelines. ${ }^{24}$ The relative core density of all test specimens was set at 0.04 as the initial relative density of 0.02 resulted in postprocessing difficulties during production. We choose to fix the height of all the specimens at $33.68 \mathrm{~mm}$ instead of following a fixed number of unit cells in any direction. The face sheet thickness was kept constant at $5 \mathrm{~mm}$ throughout. The parts were built from the bottom up with the face sheet placed orthogonally to the built direction to minimize the effects of the layer-by-layer manufacturing process, such as anisotropic properties of the completed parts, during testing. The completed parts are shown in Fig. 4.

\section{Experiment}

Compression test is a form of mechanical testing that is performed to determine the properties of the material when being compressed. In this test, fundamental parameters that determine the specimen behavior under a compressive load are measured. These include the young's modulus and yield point where feasible.

Only one sample of each structure was fabricated and tested therefore it is not possible to examine the effects of process variations in the Objet AM process or identify potential outliers. However, results arising from the study of process variations in different AM processes are well-documented. ${ }^{25}$ In the fused deposition modeling (FDM) process, which is a polymer-based AM process, the compressive strength of FDM parts was not affected by build direction as compared with injection molded parts even though small imperfection like voids, warpage, etc. may be present. Therefore, it is suggested that the Objet process may be similarly immune given that the underlying principles of both processes are very much the same.

\subsection{Test Procedure}

The standard compression tests were carried out at a feed rate of $0.1 \mathrm{~mm} / \mathrm{min}$ using an Instron 5900 Tabletop Universal Testing System. All 3 different test specimens were tested under the same conditions and experimental parameters.

The test procedures were as follows:

1. Set the parameters of the experiment

2. Place the compression test specimen at the bottom plate of the testing machine

3. Adjust/Lower the upper plate towards upper surface of the test specimen such that the specimen is firmly held

4. Preset zero the load on the measuring scale and carry out the testing

5. Record the load and extension for the construction of stressstrain curve of each specimen

6. Continue the testing until structure registered the first peak and fails

At the end of experiment, a stress-strain curve was generated for comparison. The next section will be discussed the comparison results and applications in details.

\section{Results, Discussion, and Applications}

The proposed three lattice structures were tested for their compression strength. The resulting stress-strain curves were plotted using the experimental data and shown in Fig. 5. It can be seen that the Kagome structure is able to withstand the highest compression stress of $0.65 \mathrm{MPa}$ at a strain of $3.8 \%$ before it starts to soften. For the $3 \mathrm{D}$ pyramidal, it reached a comparatively lower stress of $0.2 \mathrm{MPa}$ before softening while the hexagonal diamond structure did not exhibit any appreciable softening even at strains of more than $8 \%$ - the corresponding stress being approximately $0.1 \mathrm{MPa}$. Unlike the other structures, the test of the hexagonal diamond was stopped before the onset of plastic buckling at $9 \%$ strain. With the exception of the hexagonal diamond structure, the onset of plastic buckling is evident in all the structures tested.

To verify the validity of the results, the parameters of the compression test were converted to non-dimensional units consisting of compressive

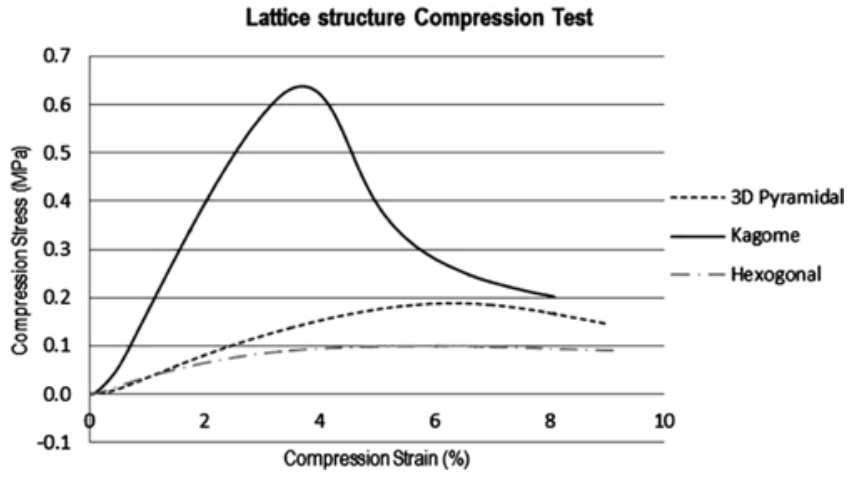

Fig. 5 Stress-strain curves of the compression test

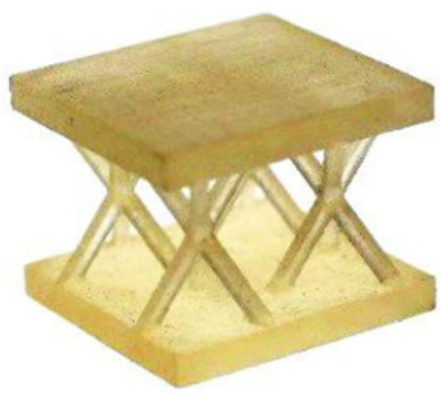

(a) 3D Kagome structure

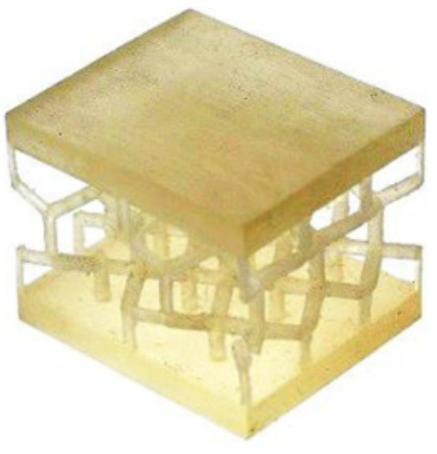

(b) Hexagonal diamond structure

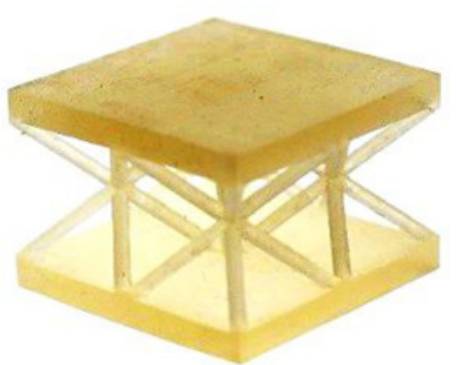

(c) 3D pyramidal structure

Fig. 4 The proposed 3D printed periodic lattice structures 
force $\left(\mathrm{F}=\mathrm{P} / \sigma_{\mathrm{y}} \mathrm{A}_{\mathrm{c}}\right)$ and strain $(\varepsilon=\Delta \mathrm{H} / \mathrm{H})$ as shown in Fig. 6 for comparison with the established results. ${ }^{16}$ The maximum load experienced by the plastic AM Kagome structure reached 5.5 whereas the maximum load experienced by the $\mathrm{Cu}$ alloy Kagome structure reached 2.6. The larger value obtained in the AM part has yet to be explained.

The research started with high expectations that the hexagonal diamond structure can compete with the Kagome structure as both are inspired by nature. Evidently, this is not the case since the results showed that the hexagonal diamond structure is not suited for withstanding compressive loads despite having demonstrated that as an atomic arrangement, it is even harder than the cubic diamond.

Nevertheless, the unique stress/strain behavior of the hexagonal diamond structure can be utilized elsewhere as energy absorbers for example. The extended section of the stress/strain curve defines the behavior of an ideal energy absorber. ${ }^{26}$ Recall that the area under the stress/strain curve represents the work done on a specimen having unit dimensions throughout. Thus, in an actual block of material having finite size this would be represented as the product of force and distance or work done. And the results are the same as kinetic energy.

Thus, the results from compressing the lattice structure will be equivalent to the kinetic energy of an object impacting the structure. When the lattice structure is designed with the appropriate size and material, it can be used to absorb all the kinetic energy of an impacting body. Most importantly, the structure that is protected by the lattice structure would not be exposed to forces higher than the maximum compressive stress of the lattice structure. The same principles apply to car bumpers and crash zones that are compressed at a constant force

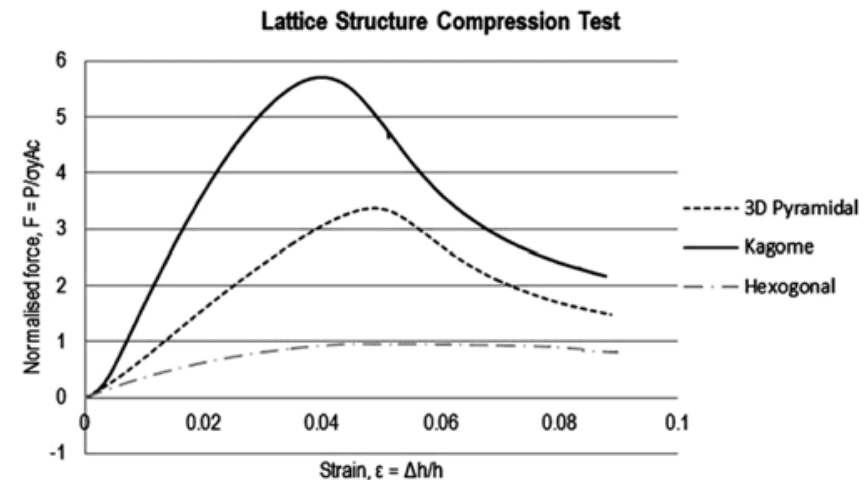

Fig. 6 Non-dimensional force/strain curves calculated in compression for Hexagonal diamond and Kagome cores

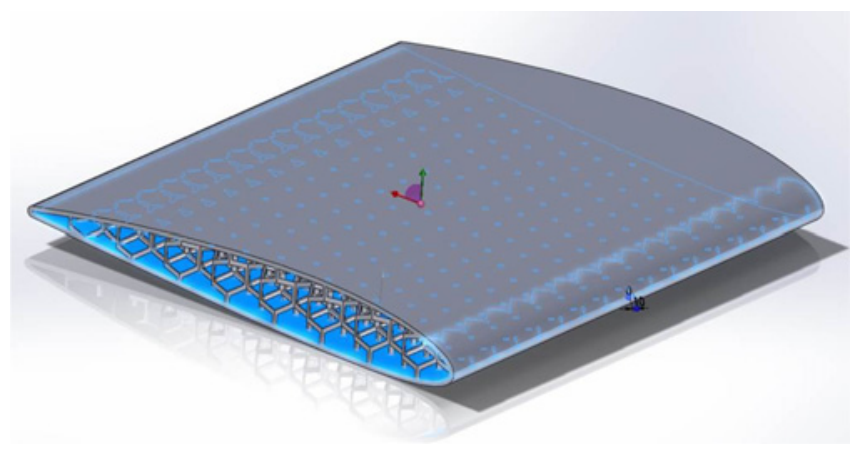

Fig. 7 The proposed CAD model of wing with light-weight highstrength reinforcement under impact to eliminate or minimize injury to its passengers.

Based on the results from the experiments, a CAD model of the aerofoil was established. The design was modified with a groove at the open end of the aerofoil to allow the attachment of the aerofoil unto the fuselage. The proposed CAD model is as shown in Fig. 7.

The deployment of the wing can occur immediately after launch and preferably takes at most one second to complete since an UAV is designed to be launched from a tube. The wing should be light and strong since the small UAV has limited power. The strength and stiffness of the inflatable wing can be maximized by controlling the material depending on the wing design, the profile of the wing, and the internal pressure. To demonstrate the usefulness of the proposed structure design, a segment of the proposed wing design was developed by the Objet $3 \mathrm{D}$ printer as shown in Fig. 8 . We selected a method to fabricate the proposed CAD model, which was commercialized by Objet Geometries Ltd. It combines ink-jet technologies with the use of photopolymers as raw materials to produce 3D parts. We used the Objet $3503 \mathrm{D}$ printer, while the selected photopolymer was a rubber-like material called Objet TangoGray FLX950. The material properties are listed in Table 2.

Through the experiments, we demonstrated that the proposed physical model can evaluate the suitability of the manufacturing process and materials based on the properties of various structure designs. Therefore, the proposed $3 \mathrm{D}$ printed evaluation process can provide with an efficient way to identify light-weight deployable structures in UAV wing design. Designers also gain insight into how the properties of the light-weight deployable structures affect design performance in UAV wings.

\section{Closing Remarks and Future Work}

In this paper, we extended the concepts of compliant mechanisms into inflatable wing design for small and deployable UAVs. The apparent strengths of truss lattices were investigated to identify the truss lattice with the optimal elastic performance according to the Hashin \& Shtrikman bounds. 3D Kagome lattice, Hexagonal diamond, and Cross pyramidal structures were used to compare the compressive strength of their lattice structures that have high values in elastic performance. The results confirmed that the 3D Kagome has the

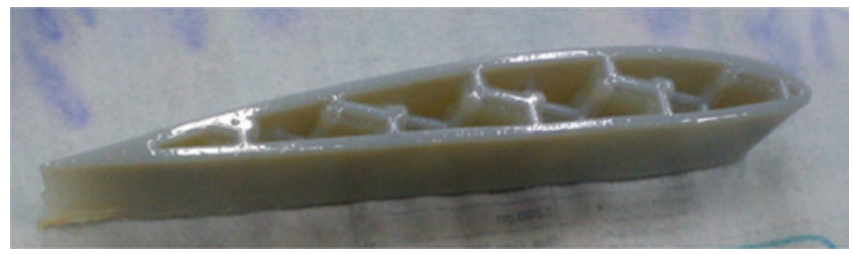

Fig. 8 A Physical Model of the Proposed Wing Design

Table 2 Properties of Objet TangoGray FLX950

\begin{tabular}{ccc}
\hline & Units & Metric \\
\hline Tensile strength & $\mathrm{MPa}$ & $3-5$ \\
\hline Elongation at break & $\%$ & $45-55$ \\
\hline Polymerized density & $\mathrm{g} / \mathrm{cm}^{3}$ & $1.14-1.15$ \\
\hline
\end{tabular}


highest load capacity although it has also been noted that the hexagonal diamond exhibited the ideal properties of energy absorbers. To demonstrate and validate the usefulness of the proposed inflatable design, we developed a prototype wing segment for UAVs using 3D printing technologies.

Future work will include investigations into other properties of the structures such as tensile and shear strengths including numerical simulations. Also, the structures will be extended to at least 5-7 unit cells in each direction to minimize the effects of boundary conditions.

\section{ACKNOWLEDGEMENT}

This study was finally supported by a start-up grant from Nanyang Technological University and an AcRF Tier 1 grant from Ministry of Education, and Seoul National University of Science \& Technology

\section{REFERENCES}

1. Cocke, B. W., "Wind-tunnel Investigation of the Aerodynamic and Structural Deflection Characteristics of the Goodyear Inflatoplane," National Advisory Committee for Aeronautics, 1958.

2. Murray, J., Pahle, J., Thornton, S., Frackowiak, T., Mello, J., et al., "Ground and Flight Evaluation of a Small-scale Inflatable-Winged Aircraft, Aiaa 2002-0820," Proc. of the 40th AIAA Aerospace Sciences Meeting \& Exhibit, 2005.

3. Park, J. H. and Kim, K. J., "Optimal Design of Camber Link Component for Light Weight Automobile using CAE(Computer Aided Engineering)," Int. J. Precis. Eng. Manuf., Vol. 14, No. 8, pp. 1433-1437, 2013.

4. Dornfeld, D. A., "Moving Towards Green and Sustainable Manufacturing,” Int. J. Precis. Eng. Manuf. Green Tech., Vol. 1, No. 1, pp. 63-66, 2014.

5. Bitzer, T., "Honeycomb Technology: Materials, Design, Manufacturing, Applications and Testing," Springer, 1997.

6. Ashby, M. F., Evans, N. A., Flack, L. J., Hutchinson, J. W., and Wadley, H. N. G., "Metal Foams: A Design Guide," ButterworthHeinemann, pp. 1-251, 2000.

7. Wadley, H. N. G., "Multifunctional Periodic Cellular Metals," Philosophical Transactions of the Royal Society A: Mathematical, Physical and Engineering Sciences, Vol. 364, No. 1838, pp. 31-68, 2006.

8. Wohlers, T. T., "Wohlers Report 2008: State of the Industry," Wohlers Associates Inc., 2008.

9. Chua, C. K., "3-dimensional Rapid Prototyping Technologies and Key Development Areas," Computing \& Control Engineering Journal, Vol. 5, No. 4, pp. 200-206, 1994.

10. Hashin, Z. and Shtrikman, S., "A Variational Approach to the Theory of the Elastic Behaviour of Multiphase Materials," Journal of the
Mechanics and Physics of Solid, Vol. 11, No. 2, pp. 127-140, 1963.

11. Francfort, G. and Murat, F., "Homogenization and Optimal Bounds in Linear Elasticity," Archive for Rational Mechanics and Analysis, Vol. 94, No.4, pp. 307-334, 1986.

12. Bhat, B. T. and Wang, T. G., "A Comparison of Mechanical Properties of Some Foams and Honeycombs," Journal of Material Science, Vol. 25, No. 12, pp. 5157-5162, 1990.

13. Wicks, N. and Hutchinson, J. W., "Optimal Truss Plates," International Journal of Solids and Structures, Vol. 38, No. 30, pp. 5165-5183, 2001.

14. Hyun, S. and Torquato, S., "Optimal and Manufacturable Twodimensional, Kagome-like Cellular Solids,” Journal of Materials Research, Vol. 17, No. 1, pp. 137-144, 2002.

15. Hyun, S., Karlsson, A. M., Torquato, S., and Evans, A. G., "Simulated Properties of Kagomé and Tetragonal Truss Core Panels," International Journal of Solids and Structures, Vol. 40, No.25, pp. 6989-6998, 2003.

16. Wang, J., Evans, A. G., Dharmasena, K., Wadley H. N. G., "On the Performance of Truss Panels with Kagome Cores," International Journal of Solids and Structures, Vol. 40, No. 25, pp. 6981-6988, 2003.

17. Hanneman, R. E., Strong, H. M., and Bundy, F. P., "Hexagonal Diamonds in Meteorites: Implications," Science, Vol. 155, No. 3765, pp. 995-997, 1967.

18. Bundy, F. P. and Kasper, J. S., "Hexagonal diamond-A New Form of Carbon,” Journal of Chemical Physics, Vol. 46, p.3437-3446 1967.

19. Pan, Z., Sun, H., Zhang, Y., and Chen, C., "Harder than Diamond: Superior Indentation Strength of Wurtzite BN and Lonsdaleite," Physical Review Letters, Vol. 102, No. 5, Paper No. 55503, 2009.

20. Chua, C. K., Leong, K. F., and Lim, C. S., "Rapid Prototyping: Principles and Applications," World Scientific Publishing Company, 3rd Ed., 2010.

21. Gothait, H., "Apparatus and Method for Three Dimensional Model Printing," US Patent, No 6259962 B1, 2001.

22. Gothait, H., "System and Method for Three Dimensional Model Printing," US Patent, No 6658314 B1, 2003.

23. Gothait, H., "System and Method for Three Dimensional Model Printing," US Patent, No 6850334 B1, 2005.

24. Wadley, H. N., "Multifunctional Periodic Cellular Metals," Philosophical Transactions of the Royal Society A: Mathematical, Physical and Engineering Sciences, Vol. 364, No. 1838, pp. 31-68, 2006.

25. Chu, W. S., Kim, C. S., Lee, H. T., Choi, J. O., Park, J. I., et al., "Hybrid Manufacturing in Micro/Nano Scale: A Review," Int. J. Precis. Eng. Manuf. Green Tech., Vol. 1, No. 1, pp. 75-92, 2014.

26. Puglsey, A., "The Crumpling of Tubular Structures under Impact Conditions," Proc. of the Symposium on the Use of Aluminum in Railway Rolling Stock, pp. 22-41, 1960. 